Any meso-chlorins that might be formed at this stage would be re-oxidized to meso-porphyrins because of their extremely low redox-potential. Only much later, after complete reduction of all carbonyl-groups, can the porphyrin-system be partially reduced with formation of the fossil chlorins.

A more detailed report of this work is being prepared for publication elsewhere. This investigation has been supported by a research contract with the United States Office of Naval Research (Contract NONR-2196 (00)) and the grant of a summer fellowship to one of us (G. O.).

\section{Max Blumer} GrLbert OMEnN

Woods Hole Oceanographic Institution, Woods Hole, Mass.

${ }^{1}$ Treibs, A., Ann., 517, 172 (1935); Angew. Chem., 49, 682 (1936). ${ }^{2}$ Blumer, M., Helv., 33, 1627 (1950).

${ }^{3}$ Stern, A., and Pruckner, F., Z. phys. Chem., A, 180, 321 (1937).

${ }^{4}$ Blumer, M., Anal. Chem., 28, 1640 (1956).

\section{PHYSICS}

\section{Iron-55 from Nuclear Detonations}

$\mathrm{IT}_{\mathrm{T}}$ is well known that the large numbers of neutrons released during nuclear bomb detonations $(\sim 0.5$ $\mathrm{kgm}$. neutrons per megaton explosion $)^{1}$ can induce high levels of radioactivities in the hardware and associated structures of the apparatus. Of particular interest is iron-55, an X-ray emitter with a half-life of $2 \cdot 6$ years, formed by $(n, 2 n)$ and $(n, \gamma)$ reactions on iron-56 and iron-54, respectively.

The site of high specific activity of iron-55 should be in the marine environment where stable iron exists in very low concentrations ( $\sim 10 \mu \mathrm{gm} . / 1$. sea water). Radioactive iron falling on continental surfaces will be diluted readily in the large amounts of iron already existing there.

We thought it desirable to ascertain the present content of iron-55 in the oceans. Although this can be accomplished by measuring the vertical depth profiles of iron -55 contents at a number of marine locations, a more expeditious approach is the isolation of the iron activity from organisms that concentrate this element from the oceanic environment. The tuna fish which inhabit both the deep and shallow waters of the seas seemed especially suitable for this purpose since they may be considered to sample rather large areas because of their extensive migratory habits.

Ten kilograms of livers and hearts of tuna fish collected from the Pacific Ocean were digested with nitric and perchloric acids. The residue was dissolved in $8 \mathrm{~N}$ hydrochloric acid and the iron extracted with isopropyl ether. Further purification was achieved by fixing the iron on an anion exchange resin. The amount of elemental iron recovered was $2 \cdot 5 \mathrm{gm}$.

The sample was assayed in a proportional counter shielded by $8 \mathrm{in}$. of iron and with an appropriate anticoincidence arrangement. The purity of the activity was determined by comparing the spectrum of emitted X-rays with that of a standard iron-55 source. The specific activity of the samples, in February 1961 , was found to be $82,000 \mathrm{~d}$.p.m./gm. of iron. This value may be compared with those of other artificial and natural isotopes in the marine environment: strontium-90, 10 d.p.m./gm. strontium ; cæsium-137, 500,000 d.p.m./gm. cæsium; and uranium-238, 700,000 d.p.m./gm. uranium. Since iron is generally the most abundant heavy metal in the marine biosphere, the activity-level of iron-55 is probably the highest. It should be noted that it is not great enough to constitute a health hazard.

An approximation of the iron-55 inventory in the Pacific may be obtained from the observed average specific activity of the oceanic iron. Assuming a concentration of $10^{-5} \mathrm{gm}$. iron/l. of sea water and an average oceanic depth of $4,000 \mathrm{~m}$. (that is, 400 1. $\left(\mathrm{em} .{ }^{2}\right)$, the iron-55 inventory in the Pacific should be of the order of $82,000 \times 10^{-5} \times 400$ or 330 d.p.m. $/ \mathrm{cm} .^{2}$ of oceanic area. Most of this activity probably still resides in the upper water inasmuch as the marine biosphere tends to promote an accumulation of iron in the oceanic mixed layer ${ }^{2}$.

An attempt was made to find out whether any significant fraction of the iron -55 activity had already settled to the sea floor. The upper $1 \mathrm{~cm}$. of sediment layer was taken from two cores of total cross-sectional area of $40 \mathrm{~cm} .{ }^{2}$, raised in the Pacific $\left(28^{\circ} 58^{\prime} \mathrm{N}\right.$. ; $117^{\circ} 28^{\prime} \mathrm{W}$.; 3,600 m. depth) in April 1961. The sample was leached with $6 N$ hydrochloric acid and the iron in solution was extracted and purified as described above. The iron-55 activity in the entire sample was very small ( $\leq 3$ d.p.m.), implying that only an insignificant fraction of the activity had settled to the ocean bottom during the past few years.

In order to obtain a measure of atmospheric fall-out to the oceans, rain from San Diego was assayed for iron-55 activity. It yielded a value of 0.8 d.p.m./l. in February 1961. This indicates that the present contribution of atmospheric fall-out is small compared with the total inventory in the ocean. One metre of rainfall introduces only about 0.08 d.p.m. of iron-55 annually per cm. ${ }^{2}$ of oceanic area.

Acknowledgment is made to the Inter-American Tropical Tuna Commission for aid in obtaining the fish-livers. This work was sponsored by the Division of Biology and Medicine, U.S. Atomic Energy Commission.

RAMA*

MINORU KOIDF

Edward D. Goldberg†

Department of Earth Sciences,

University of California, San Diego.

* On leave from the Tata Institute of Fundamental Research, Bombay.

$\dagger$ Present address: Institute of Physics, University of Bern, Switzerland.

${ }^{1}$ Libby, W. F., Science, 123, 657 (1956).

${ }^{2}$ Lewis, G. S., and Goldberg, E. D., J. Marine Res., 13, 183 (1954).

\section{Quenching of Cation Vacancies in Doped Crystals of Sodium Chloride}

As is well known, the thermodynamic defects developed by alkali halide crystals at high temperatures are predominantly of the Schottky type. The number of eation and anion vacancies in equilibrium with the erystal, at temperatures near the melting point, for example, may be as large as $10^{-4}$ of the total number of atoms. Attempts have, therefore, been made to quench these vacancies by very rapid cooling from these high temperatures, but without success, judging from the very similar values obtained for the electrical conductivities of the quenched and the annealed specimens ${ }^{1}$. (From the melt, however, some of the defects can be quenched.) This, however, is not surprising, since the relaxation times corresponding to the migration of vacancies, leading either to formation of pairs or to their exit from the crystal, 